\title{
Parameters Representing Muscle Tone, Elasticity and Stiffness of Biceps Brachii in Healthy Older Males: Symmetry and Within-Session Reliability Using the MyotonPRO
}

Louise Bailey, Dinesh Samuel*, Martin Warner and Maria Stokes

Faculty of Health Sciences, University of Southampton, United Kingdom

\begin{abstract}
Background: Clinical assessments of muscle tone are subjective, often using the non-affected side for comparison. The MyotonPRO offers portable, non-invasive, objective measurement of mechanical properties of muscles.

Objective: This study aimed to investigate between-limb symmetry for mechanical properties of biceps brachii (BB) in older males and within-session intra-rater reliability of a novice user of the MyotonPRO device.

Methods: Twenty community-dwelling, right-handed males aged 65-85 years (mean 71.7; SD \pm 4.9 ) were studied. Exclusion criteria: history of neurological and musculoskeletal conditions, medications affecting muscle tone, and body mass index $>30 \mathrm{~kg} / \mathrm{m}^{2}$. The device applied 10 mechanical impulses at one second intervals, producing damped oscillations, from which frequency (non-neural tone), stiffness and logarithmic decrement (elasticity) were measured. With the participant resting supine, two consecutive sets of 10 recordings were taken bilaterally from BB.

Results: Percentage mean differences between-sides for larger and smaller values were 12\% (tone), $14 \%$ (stiffness) and $27 \%$ (elasticity), which were statistically significant $(p<0.001)$. Within-session reliability was excellent for all three parameters (ICC 3,2: all 0.99). Bland and Altman plots confirmed good agreement, without bias.

Conclusion: Symmetry of BB mechanical properties in a group of older males was less than $15 \%$ for tone and stiffness but not elasticity. Within-session intra-rater reliability of a novice user was excellent for all three parameters. Reliability over different days needs to be investigated. These findings indicate potential clinical application of the MyotonPRO for assessing abnormalities of muscle parameters in patients with neurological or musculoskeletal conditions, using comparison with the contralateral side (absolute difference), as well as databases of normative reference values from healthy control groups of different ages, gender and activity levels.
\end{abstract}

Keywords: Biceps brachii; Muscle tone; Mechanical properties; Reliability; Symmetry

\section{Introduction}

Stroke is common in older people, with those over the age of 60 years being at greatest risk [1]. The most common muscular impairment following stroke is spasticity, defined as "a motor disorder characterized by a velocity-dependent increase in tonic stretch reflexes (muscle tone) with exaggerated tendon jerks" [2]. Treatment of spasticity, or increased tone, underpins the rehabilitation aims in the stroke clinic [3], but objective assessment of muscle tone is not currently possible in the clinical setting.

Clinical measures of tone are subjective, allowing limited comparison with population normal values or accurate monitoring of medical or therapeutic treatment efficacy. Widely used scales are the Modified Ashworth Scale [4] and the Tardieu Scale [5], lack temporal and inter-rater reliability [6]. Bilateral muscular symmetry is assumed through palpation of resistance of the muscle to continuous passive stretch as compared to the contralateral side [4]. Crucially in hemi-paretic patients, objective measures of tone are required for quantitative investigation of therapeutic interventions. New measures of muscular properties are being developed, offering an in vivo, objective, clinical, non-invasive alternative to subjective assessments. These include tensiomyography or TMG, which uses electrical stimulation to elicit muscle oscillations to measure contraction time and muscle displacement [7]; various devices that measure tissue stiffness, such as muscle 'hardness' meters [8], myotonometry, which compresses ( $\geq 1 \leq 2$ seconds) the muscle to record tissue displacement $[9,10]$, and a mechano-acoustic indentor system that records tissue displacement to measure non-linear elastic properties [11]; and the recently introduced Myoton technology, which elicits mechanically induced oscillations [12-21]. The different technologies have their relative advantages. Myoton devices lend themselves readily to clinical testing, as they are compact and hand-held, measure various parameters and do not need to be connected to a computer during data collection.

Myoton devices apply mechanical impulses, producing damped oscillations, from which several muscle parameters can be computed simultaneously; those most commonly documented are frequency (non-neural tone), logarithmic decrement (indicating elasticity), and stiffness [12]. Muscle tone is not strictly a mechanical property but a state of tension. The MyotonPRO is a novel hand-held device (Myoton AS, Estonia and Myoton Ltd, London) and earlier prototypes have contributed to existing literature, with the Myoton- 2 and 3 being the most documented (see below). The present study is amongst the first to use the MyotonPRO device $[13,14]$. Although the basic principle of testing remains the same, some differences are detailed in the Methods

*Corresponding author: Dr Dinesh Samuel, Faculty of Health Sciences, Building 45, University of Southampton, Highfield Campus, Southampton, SO17 1BJ, United Kingdom, Tel: +44 (0)2380 598925; E-mail: D.Samuel@soton.ac.uk

Received February 21, 2013; Accepted April 12, 2013; Published April 15, 2013

Citation: Bailey L, Samuel D, Warner M, Stokes M (2013) Parameters Representing Muscle Tone, Elasticity and Stiffness of Biceps Brachii in Healthy Older Males: Symmetry and Within-Session Reliability Using the MyotonPRO. J Neurol Disord 1: 116. doi:10.4172/2329-6895.1000116

Copyright: @ 2013 Bailey L, et al. This is an open-access article distributed under the terms of the Creative Commons Attribution License, which permits unrestricted use, distribution, and reproduction in any medium, provided the original author and source are credited. 
section. Myoton technology has been examined for the assessment of muscle in neurological conditions, including Parkinson's disease [15] and stroke [16,17]. The technology is potentially useful for aiding assessment of muscle dysfunction and monitoring the effects of treatment on muscle tone and rigidity (elasticity), which are important impairments in the case of stroke and Parkinson's disease respectively. Reference values from healthy cohorts are needed for comparison in such studies.

Reliability of using prototypes of Myoton devices has generally been good but varied with the muscle studied [17-21]. When compared with TMG, the Myoton-3 device was more reliable on repeated testing and more valid for detecting changes in muscle parameters at different muscle lengths [20]. The within day (sessions 30 minutes apart) intra-rater reliability of the Myoton-3 in BB in a small group of stroke patients $(n=12)$ produced fair to good ICCs for both the affected side (ICC: 0.54-0.99) and poor to good for the non-affected sides (ICC 0.25- 0.98) [16]. A larger study of 62 stroke patients, testing 60 minutes apart, found excellent ICCs for BB and triceps in both limbs (ICC 0.79-0.96) apart from BB tone in the unaffected limb (ICC=0.72) [17]. Reliability of novice user is important to examine, not only to reflect the clinical situation but as it may influence the likely uptake of the technique by clinicians if it is found to be easy to use.

The contralateral side is often used clinically to assess abnormalities of the injured side [22,23]. Such comparison requires knowledge of the normal level of symmetry. Myoton studies on stroke patients have not compared data with healthy control data to examine the magnitude of asymmetry as a guide to the degree of abnormality. Studies of muscles in healthy older populations are lacking and therefore needed for comparison with patient groups, in terms of providing reference values, establishing normal symmetry and assessing reliability.

Biceps brachii (BB) is an important muscle to test in older people. With approximately half of stroke survivors experiencing difficulty with a non-functional arm [3], the BB muscle often forms the focus of rehabilitation efforts. Between-side symmetry of mechanical properties has not been established for healthy older adults.

\section{Aims}

The present study aimed to: 1) investigate the level of betweenside symmetry between $\mathrm{BB}$ muscle characteristics of the two upper limbs measured using the MyotonPRO in older males over the age of 65 years and, 2) establish the within-session intra-rater reliability of a novice user of the MyotonPRO device.

\section{Material and Methods}

\section{Participants}

Twenty healthy, right-hand dominant, community-dwelling older males (aged 65-85 years; mean 71.7) were recruited via posters in local community areas and presentations in social groups. A sample of 20 participants is recommended as sufficient for reliability studies $[24,25]$. Information on handedness, determined using the Edinburgh Handedness Inventory [26], and levels of physical activity, determined by the validated Physical Activity Scale for the Elderly (PASE) [27] were obtained during telephone screening. Exclusion criteria included being left-handed or ambidextrous, having medical conditions or medications known to affect muscle tone, having a BMI $>30 \mathrm{~kg} / \mathrm{m}^{2}$ which may cause oscillations to be too attenuated to measure [28], or a current upper limb injury or skin condition over the BB muscle where the testing device was to be applied.

\section{Measurement of mechanical parameters}

Myoton technology is well described in the literature [20], with the MyotonPRO being the latest and more compact version of the device, which is not affected by gravity [14]. This is achieved by the device using a triaxial accelerometer and a system which allows the device to be held in any direction when taking measurements, thus making muscles more accessible in different postures. However, as muscle tissue is affected by gravity, it is recommended that muscles are placed at less than 90 degrees to the horizontal. The device is held perpendicular to the skin surface, so that the mechanical impulse is delivered to the muscle, eliciting effective damped oscillations. The device performs simultaneous computation of the tissue parameters, and the three measured in the present study were its non-neural tone (state of resting tension or the muscle's resistance to passive stretch during resting state) represented by frequency of oscillation $(\mathrm{Hz})$, and mechanical properties of stiffness $(\mathrm{N} / \mathrm{m})$, or rigidity of tissue indicating the ability to resist force that modifies its shape, and elasticity (logarithmic decrement), indicating the muscle's ability to recover its shape after being deformed (Figure 1). Frequency is defined as the maximum frequency $\left(\mathrm{F}=\mathrm{f}_{\max }\right)$ computed from the signal spectrum by FFT-Fast Fourier Transform. The higher the frequency of the dampened oscillations (natural oscillation frequency), the higher is the tone (intrinsic state of resting tension without voluntary contraction). Frequency is known to increase with contraction (state of tension) [12] and stretch [20]. Stiffness is calculated as

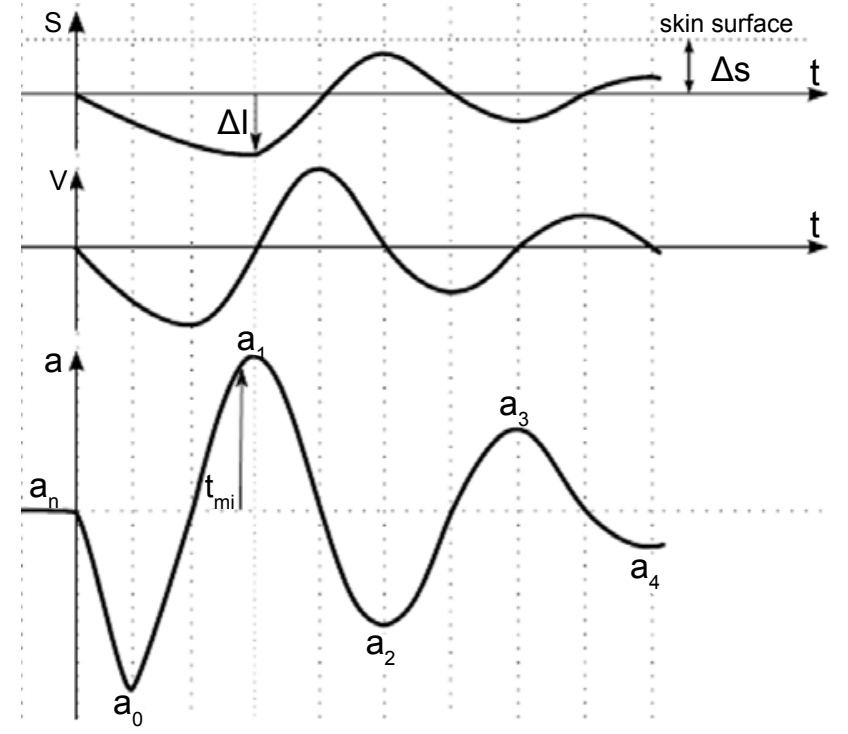

$\Delta \mathrm{S}$ : Pre-compression of subcutaneous tissues above muscle being measured; $\Delta \mathrm{l}$ : Maximum displacement of the tissue; S: Displacement, tissue oscillation $(\mathrm{mm})$; V: Oscillation velocity $(\mathrm{m} / \mathrm{s})$; a: Acceleration of oscillation $(\mathrm{mG})$; t:Time (ms); $a_{0}$ : Maximum acceleration; $t_{m i}$ : End of mechanical impulse; $a_{1}$ : Maximum acceleration representing the maximum displacement of the tissue i.e. maximum tissue resistance $(\mathrm{mG}) ; a_{2}$ : Maximum acceleration at the point of opposite displacement due to residual inertia of the tissue oscillation; $a_{3}$ : Maximum acceleration of the second period of oscillation-occurs due to recuperation of stored residual mechanical energy in the tissue.

Figure 1: Waveforms illustrating muscle oscillation and its relative displacement $(\mathrm{S})$, velocity $(\mathrm{V})$ and acceleration (a). The purpose of the figure is to describe the muscle oscillation i.e. displacement (S), velocity of the oscillation (V) in relation to the oscillation acceleration (a). When the mechanical impulse is delivered and released quickly under constant pre-compression, the muscle responds immediately in the form of a damped oscillation, causing co-oscillation of: the pre-compressed subcutaneous tissue layers above the muscle; the testing-end; measurement mechanism; and accelerometer attached to the measurement mechanism. (Diagram provided courtesy of Myoton AS, Estonia). 
Citation: Bailey L, Samuel D, Warner M, Stokes M (2013) Parameters Representing Muscle Tone, Elasticity and Stiffness of Biceps Brachii in Healthy Older Males: Symmetry and Within-Session Reliability Using the MyotonPRO. J Neurol Disord 1: 116. doi:10.4172/2329-6895.1000116

Page 3 of 7

$S=m_{\text {probe }}\left(\frac{a_{\max }}{\Delta l}\right)$ and the higher the $\mathrm{N} / \mathrm{m}$ value, the stiffer the muscle is [12]. Elasticity, characterised by logarithmic decrement of the dampened oscillations, is expressed in arbitrary units $\left[\theta=\ln \left(\frac{a_{1}}{a_{3}}\right)\right]$ and indicates how much mechanical energy is lost in the tissue during an oscillation cycle. The smaller the decrement value, the smaller will be the dissipation of mechanical energy and higher will the elasticity of a tissue [12]. Decrement of zero would represent absolute elasticity and zero dissipation of mechanical energy (Figure 1).

\section{Experimental procedure}

Prior to data collection, participants were asked to refrain from strenuous physical activity for at least 48 hours, and alcohol for at least 24 hours. Test protocols were designed so they could be replicated easily in the clinic. Participants were positioned in supine lying with pillows supporting their forearms in neutral anatomical alignment. A rolled towel under the wrist produced approximately 10-15 degrees elbow flexion to release stretch on $\mathrm{BB}$. The testing site on the muscle belly was then located using a tape measure to identify the point midway between the anterior aspect of the lateral tip of the acromion and the medial border of the cubital fossa (Figure 2), and marked on the skin using a non-toxic non-permanent marker. Modification of this protocol for locating the testing site is suggested in the discussion section but was not followed in the present study.

A 10-minute rest enabled the baseline relaxation state of the muscle to be achieved prior to testing. The tip of the probe (or testing end; $3 \mathrm{~mm}$ diameter) was applied to the skin perpendicular to its surface over the muscle of interest at a constant pre-load ( 0.18 Newtons) to pre-compress subcutaneous tissues. An automatic system triggered a short electromagnetic impulse with constant force ( 0.4 Newtons) that was transmitted to the tissue, applying a brief mechanical impulse (15 milliseconds) with quick release (http://www.myoton.com/en/ Technology/Technical-specification). The device was used in multiscan mode consisting of a set of 10 mechanical impulses, one second apart. Values for mean, standard deviation and coefficient of variation (CV) for the 10 measurements, i.e. one measurement set, appear on the device's screen [12]. If the CV of a set exceeded $3 \%$, as displayed on
A

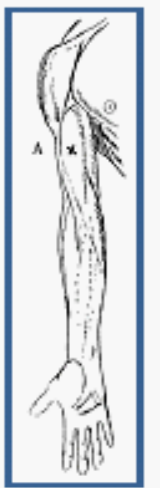

B

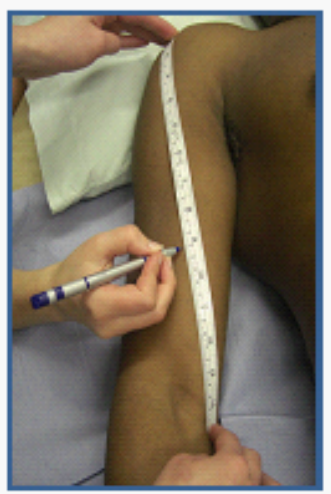

C

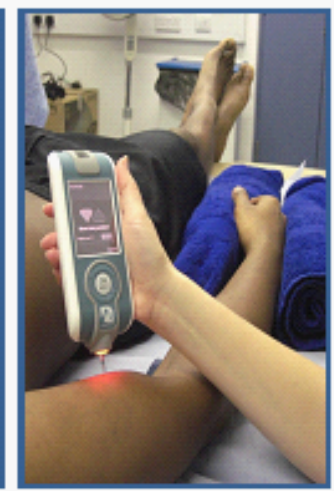

Figure 2: Location of testing site for biceps brachii. The equidistant point between the anterior aspect of the lateral tip of the acromion and the medial border of the cubital fossa (A: (10)) was located using a tape measure and marked on the skin with non-toxic washable ink pen (B). The MyotonPRO was then applied perpendicularly to the skin surface $(C)$. the screen, the set was erased and re-measured. This criterion aimed to maximise consistency on the part of the investigator and did not need to be employed frequently. Two sets of 10 impulses were recorded on both the dominant and non-dominant sides, and the mean of each set used in the analysis. The operator (lead investigator, LB), a novice user who had been formally trained for four hours by Myoton Ltd, and undertook a further one day's practice, performed the measurements. Right BB was always tested first for ease of data management and standardisation across participants.

\section{Statistical analysis}

MyotonPRO readings were imported into Microsoft Excel. SPSS Statistics v19 was used for data analysis. Data were examined for normality using the Shapiro-Wilks test and frequency histograms. A confidence interval of $95 \%$ allowed significance of $\mathrm{p}<0.05$.

Descriptive statistics (mean, SD and range) were calculated bilaterally for each parameter. The mean of the dominant (right) $\mathrm{BB}$ was compared to the mean of the non-dominant $\mathrm{BB}$. The actual difference (non-dominant subtracted from dominant side) between limbs was calculated to assess symmetry. To examine whether true differences between sides were masked in the analysis considering dominance, the limb with the largest value was compared to the limb with the smallest value for each parameter. This calculation provides the absolute difference, which could also be calculated using dominance but taking the non-negative value of the actual difference for each participant and then calculating the mean. Percentage differences using the actual (dominant versus nondominant) and absolute (larger versus smaller) differences divided by the mean values, (multiplied by 100) were also calculated. Paired $\mathrm{t}$-tests were used to examine differences between sides for both types of comparison (dominant versus non-dominant and larger versus smaller), to examine whether the former was appropriate and did not mask true differences within the group mean data.

Analysis of reliability used intra-class correlation coefficients (ICCs) for each parameter between the two sets of measurements, using an average measures ICC $(3,2)$ model.

The following classification was used for interpreting level of reliability from ICCs: Excellent $>0.75$; Good to Fair=0.74-0.4; Poor $<0.4$ [29]. In order to determine any meaningful differences from data error (precision), the standard error of measurement (SEM) was calculated by: $S E M=S D x(\sqrt{1-I C C})$, where $S D$ is the pooled standard deviation, and the ICC is the reliability coefficient [30].

Bland-Altman analysis assessed the variability between the two sets of measurements for each parameter to determine the level of agreement [31]. The limits of agreement were calculated as the mean difference plus or minus two standard deviations of the differences between each score.

\section{Ethical considerations}

The Faculty of Health Sciences Ethics Committee, University of Southampton, approved the study. Procedures and risks of participation were explained and written informed consent was obtained from all participants.

\section{Results}

Twenty volunteers were studied and their characteristics are shown in table 1 . The data were normally distributed for all three parameters $(\mathrm{p}>0.05)$, so parametric statistics were used for analysis. 


\begin{tabular}{|l|l|l|}
\hline Baseline Data & Mean (SD) & Range \\
\hline Age (years) & $71.7(4.9)$ & $65-82$ \\
\hline Weight $(\mathrm{kg})$ & $75.6(12.9)$ & $55-98.5$ \\
\hline Height $(\mathrm{cm})$ & $1.73(0.1)$ & $1.64-1.83$ \\
\hline BMI $\left(\mathrm{kg} / \mathrm{m}^{2}\right)$ & $25.2(3.5)$ & $19.5-30$ \\
\hline No. of participants (n=20) & \\
\hline Ethnic origin (white/other) & $13 / 7$ \\
\hline Comorbidities (Diabetes/Previous TIAs) & $3 / 3$ \\
\hline Previous occupation (Active/Sedentary) & $7 / 13$ \\
\hline Taking regular medication? (Y/N) & $15 / 5$ \\
\hline Current physical activity levels (PASE) (Light/Moderate) & $5 / 15$ \\
\hline
\end{tabular}

Table 1: Baseline demographic characteristics of participants and testing conditions.

\begin{tabular}{|c|c|c|c|c|c|c|}
\hline \multicolumn{3}{|c|}{$\begin{array}{l}\text { Dominant vs. non-dominant } \\
\text { analysis } \\
\text { (Actual difference) }\end{array}$} & & & 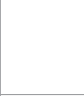 & 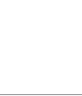 \\
\hline & \multicolumn{2}{|c|}{$\begin{array}{c}\text { Tone } \\
\text { (Frequency; Hz) }\end{array}$} & \multicolumn{2}{|c|}{ Stiffness (N/m) } & \multicolumn{2}{|c|}{$\begin{array}{l}\text { Elasticity (Log } \\
\text { Decrement) }\end{array}$} \\
\hline & D & ND & D & ND & D & ND \\
\hline Mean & 14.4 & 14.4 & 235.0 & 238.6 & 1.3 & 1.3 \\
\hline SD & 1.5 & 1.4 & 27.4 & 29.0 & 0.2 & 0.2 \\
\hline Range & $11.9-17.0$ & 12.1- 17.2 & $188.2-301.3$ & 177.7- 281.5 & $1.0-1.8$ & $1.0-1.8$ \\
\hline Actual Difference & \multicolumn{2}{|c|}{0.02} & \multicolumn{2}{|c|}{3.60} & \multicolumn{2}{|c|}{-0.04} \\
\hline \% Difference & \multicolumn{2}{|c|}{0.10} & \multicolumn{2}{|c|}{1.43} & \multicolumn{2}{|c|}{3.84} \\
\hline$\%$ SD & \multicolumn{2}{|c|}{9.61} & \multicolumn{2}{|c|}{10.59} & \multicolumn{2}{|c|}{20.80} \\
\hline$P$ valuea & \multicolumn{2}{|c|}{$p=0.94$} & \multicolumn{2}{|c|}{$p=0.53$} & \multicolumn{2}{|c|}{$p=0.48$} \\
\hline $95 \% \mathrm{Cl}$ & \multicolumn{2}{|c|}{-0.61 to 0.66} & \multicolumn{2}{|c|}{-15.52 to 8.32} & \multicolumn{2}{|c|}{-0.18 to 0.09} \\
\hline \multicolumn{7}{|c|}{$\begin{array}{l}\text { Larger vs. smaller value analysis } \\
\text { (Absolute difference) }\end{array}$} \\
\hline & L & $\mathbf{S}$ & $\mathbf{L}$ & $\mathbf{S}$ & $\mathbf{L}$ & $\mathbf{S}$ \\
\hline Mean & 15.22 & 13.61 & 252.30 & 222.28 & 1.50 & 1.19 \\
\hline \% Difference & \multicolumn{2}{|c|}{12} & \multicolumn{2}{|c|}{14} & \multicolumn{2}{|c|}{27} \\
\hline$\%$ SD & \multicolumn{2}{|c|}{7.77} & \multicolumn{2}{|c|}{8.37} & \multicolumn{2}{|c|}{15.85} \\
\hline$p$ value ${ }^{a}$ & \multicolumn{2}{|c|}{$p<0.001$} & \multicolumn{2}{|c|}{$p<0.001$} & \multicolumn{2}{|c|}{$p<0.001$} \\
\hline $95 \% \mathrm{Cl}$ & \multicolumn{2}{|c|}{-2.06 to -1.15} & \multicolumn{2}{|c|}{-38.15 to -21.90} & \multicolumn{2}{|c|}{-0.39 to -0.23} \\
\hline
\end{tabular}

aSignificance level $\mathrm{p}<0.05$; Cl: Confidence intervals; SD: Standard deviation; D: Dominant side; ND: Non-dominant side; L: side on which largest values were found; S: Smallest values

Table 2: Symmetry of dominant vs. non-dominant, and larger vs. smaller values of biceps brachii characteristics in healthy right-handed older males.

\begin{tabular}{|c|l|l|l|l|}
\hline & Tone (Frequency) & Stiffness & Elasticity \\
\hline Within-day 1 & ICC $(3,2)$ & 0.99 & 0.99 & 0.99 \\
\hline SEM & $0.09 \mathrm{~Hz}$ & $2.39 \mathrm{~N} / \mathrm{m}$ & 0.02 (log decrement) \\
\hline
\end{tabular}

aScale for interpretation: Excellent $>0.75$; Good to Fair $=0.74-0.4$; Poor $<0.4$; SEM: Standard Error of Measurement

Table 3: Reliability within-session for tone (frequency, stiffness and elasticity of biceps brachii; intraclass correlation coefficients (ICC), standard error of measurement (SEM).

\section{Muscle symmetry}

Statistical analysis indicated the percentage differences for all parameters were small $(<4 \%)$ when the dominant and non-dominant sides were compared for the group, i.e. actual difference (Table 2). A paired t-test found no significant difference between sides for, tone $(\mathrm{p}=0.94)$, stiffness $(\mathrm{p}=0.53)$ or elasticity $(\mathrm{p}=0.48)$ in this population sample. Decrement displayed the greatest difference but it was not significant $(3.8 \%)$.

When analysis was performed comparing the limb showing the larger value with the limb showing the smaller value to calculate the absolute difference, mean differences of $12 \%, 14 \%$ and $26 \%$ were found

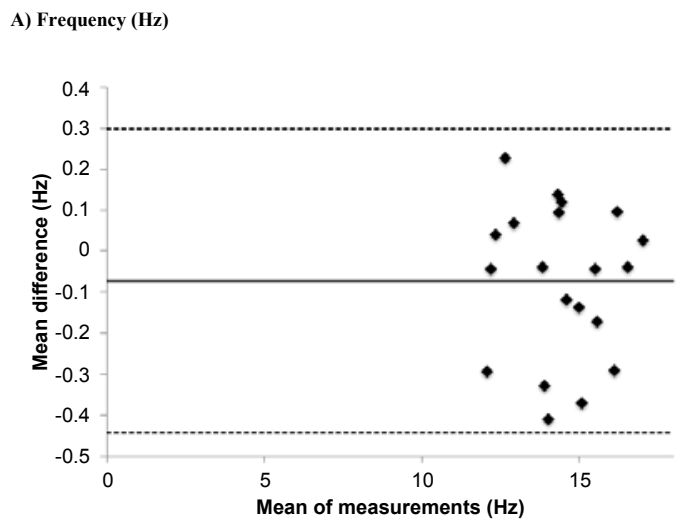

B) Stiffness (Nm)
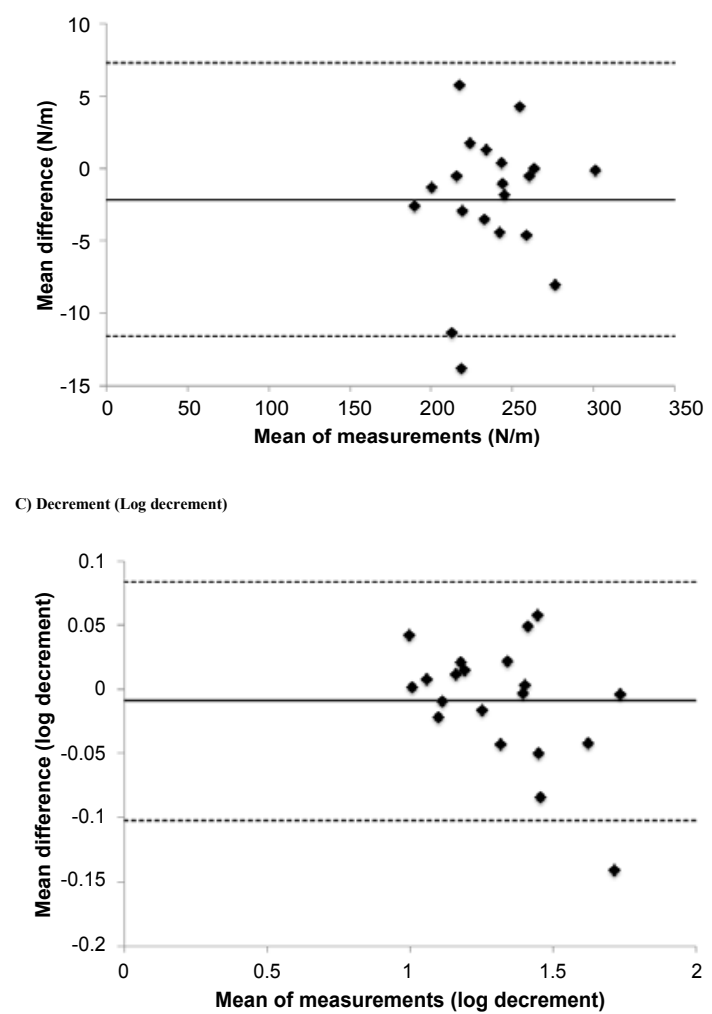

Figure 3: Bland and Altman plots highlighting the within sessiona agreement for A) frequency $(\mathrm{Hz}), \mathrm{B})$ stiffness $(\mathrm{Nm})$ and $\mathrm{C}$ ) decrement (Log decrement). The solid line represents the mean difference. The 95\% upper and lower limits of agreement are represented by the dashed line and show two standard deviations above and below the mean difference respectively. ${ }^{a}$ Using two sets of 10 measurements.

for tone, stiffness and elasticity, respectively (Table 2). The absolute differences between larger and smaller values for each parameter were found to be significant $(\mathrm{p}<0.001)$.

\section{Reliability}

Within-session reliability for all three parameters was excellent (ICC 3,2: all 0.99) and SEMs were small (Table 3).

\section{Bland-Altman analysis}

Bland Altman plots showed good intra-rater agreement in BB for the three parameters, with all points distributed evenly around zero 


\begin{tabular}{|c|c|c|c|}
\hline Parameter & $\begin{array}{c}\text { Mean Difference } \\
\text { between Raters }\end{array}$ & Upper Limit & Lower Limit \\
\hline Frequency & -0.07 & 0.30 & -0.44 \\
\hline Stiffness & -2.13 & 7.30 & -11.57 \\
\hline Decrement & -0.01 & 0.08 & -0.10 \\
\hline
\end{tabular}

Table 4: Mean difference and limits of agreement for Bland and Altman analysis, showing within-session agreement.

(Figure 3), thus indicating no bias and good levels of repeatability. The mean differences and $95 \%$ limits of agreement for Bland and Altman analysis are presented in table 4.

\section{Discussion}

The present study is the first to establish between-side symmetry of mechanical properties of BB in healthy older males using Myoton technology. The dominant limb was not consistently associated with the larger values for mechanical parameters, so assessment of symmetry by comparison of the side with the larger value versus the side with the smaller value, i.e. absolute difference, may be more appropriate than using dominance (actual difference). Reliability of repeated tests of $\mathrm{BB}$ by a novice user within the same session was excellent. Between-day reliability needs to be tested using a refined protocol, as suggested below.

\section{Between-side symmetry}

The present study demonstrated that symmetry can be influenced by how the data are analysed. Calculating actual difference according to limb dominance $(<4 \%)$ underestimated the true (absolute) difference (12-27\%) between the two sides. The mean of $12 \%$ difference for tone would be consistent with the generally accepted $10 \%$ for muscle strength differences [22], for example, but the $14 \%$ for stiffness and certainly the $27 \%$ difference for elasticity are greater. Further work needs to establish what would constitute a clinically significant difference, to determine what value would indicate abnormality. In preparing the present study protocol, limb dominance was selected as a factor in assessing symmetry due to muscle strength tending to be greater on the dominant side [22], but such an assumption for mechanical properties was not justified. The present findings challenge screening methods using limb dominance for comparing the Myoton parameters tested.

In previously published literature reporting values for the trapezius muscle in females (mean 44.2 years; SD 14.7) [19], and eight muscles, including BB, in five young (aged 18-19 years) male triathletes [12], bilateral readings were pooled. Between-side symmetry may be a useful way of assessing abnormality as result of injury. However, in stroke patients, for example, evidence suggests the presence of reduced strength and sensorimotor function of the non-hemiplegic contralateral limb compared to healthy controls [32]. Development of disuse phenomena, decreased contralateral stabilisation of the affected side [33] and ipsilateral motor damage [34] could result in a decrease in resting muscle tone of the contralateral side. It is therefore important to note that comparisons may need to be made with documented population normative values, rather than the contralateral limb within an individual, unless studies demonstrate similar data to healthy controls.

A study using the Myoton-3 device in a mixed gender stroke population (aged $54.67 \pm 10.9$ years) found muscle tone was only significantly asymmetrical in flexor carpi radialis (0.004) and not extensor digitorum (0.416) or flexor carpi ulnaris (0.760) muscles [35]. This highlights the importance of ascertaining symmetry values in healthy populations for specific muscles prior to clinical use. Statistical significance and not percentage differences were used to assess asymmetry in the stroke study [35] but the clinical significance of between-side differences in mechanical parameters needs to be determined from large studies of healthy and patient populations.

\section{Reliability}

The operator was a novice user of Myoton technology and acquaintance with the MyotonPRO device was easily established. Training was deliberately restricted to replicate the likely limited training received by clinicians prior to using new devices that are relatively simple to use.

Excellent intra-rater reliability was demonstrated (ICC 3,2: all 0.99 ) but it is stressed that this was only for tests repeated within the same session. Between-day reliability is important to establish. Initial findings using the MyotonPRO found similar within-session findings to those in the present study for rectus femoris in healthy young [14] and older [13] males. These two earlier studies also examined betweenday reliability of testing rectus femoris in young (ICC 3,1: 0.81-0.87) [14] and older (0.77-0.82) [13] males. Studies using earlier Myoton prototypes have reported high-to-very-high test re-test reliability for sessions 30 minutes apart in stroke populations [16,17]. In 61 chronic stroke patients studied using the Myoton-3, ICCs ranged from 0.82 to 0.95 and measurements of biceps had more stability and less variability between the two sessions than the other muscles studied, which were deltoid, triceps brachii, extensor digitorum, flexor carpi radialis and flexor carpi ulnaris [17]. Bizzini and Mannion [18] found within session reliability was better than between-days using the Myoton-2 and also found that reliability varied between muscles; being high for rectus femoris, biceps femoris and gastrocnemius muscles (ICCs 0.80-0.93) but low for vastus lateralis (0.4). The authors proposed that this discrepancy may have been due to difficulty in maintaining the probe in a perpendicular orientation to the muscle [18]. The MyotonPRO has a shorter probe than the earlier prototypes, which reduces the possibility of error during testing. However, other factors can influence the technique. For instance, in some older participants, the middle of BB muscle belly lay more medially and distally than in younger participants, making precision of the muscle belly difficult to ensure, and resultant deviation of testing site from the measurement protocol outlined above. The studies on stroke patients mentioned above used palpation to locate the muscle belly and found good reliability $[16,17]$, so it may not be necessary to be as precise as in the present protocol for locating the testing site. However, the reliability on different days was not assessed in the studies on stroke patients, only for sessions 30 minutes apart [16,17], so the variability over time, when muscle characteristics and recording conditions can change and influence reliability, has yet to be studied.

\section{Normative values of data for the MyotonPRO}

The present study is the first to provide normative values for muscle (non-neural) tone, stiffness and decrement of the BB in healthy older males using the MyotonPRO device.

Published BB values for stiffness in healthy females was compared to age-matched patients with Parkinson's disease (PD), where significantly higher values were recorded in PD $(203 \pm 22 \mathrm{~N} / \mathrm{m}$ vs. 192 $\pm 8 \mathrm{~N} / \mathrm{m}, \mathrm{p}=0.004$ ) [15]. The higher stiffness values obtained during the present study $(235.04 \pm 4.33 \mathrm{~N} / \mathrm{m})$ compared to the healthy controls in the study by Marusiak et al. [15], might be attributed to gender differences [36] or position of the forearm or probe application, and highlights the importance of producing definitive reference values for 
Citation: Bailey L, Samuel D, Warner M, Stokes M (2013) Parameters Representing Muscle Tone, Elasticity and Stiffness of Biceps Brachii in Healthy Older Males: Symmetry and Within-Session Reliability Using the MyotonPRO. J Neurol Disord 1: 116. doi:10.4172/2329-6895.1000116

Page 6 of 7

different healthy populations using standardised protocols, before use of the technique for routine clinical testing.

\section{Limitations of the study}

The limited size of the present convenience sample, although justified for measuring reliability [25], is not sufficient to provide reference data for this population, thus limiting its generalizability. Larger studies are recommended to produce reference data-sets allowing comparisons with populations of different age groups, genders and levels of physical activity. In addition, documentation of symmetry for different populations and muscle groups is advocated.

The experimental protocol was intended to be clinically relevant. The degree of elbow flexion was therefore only estimated and not measured. The elbow angle used in the present study (approximately 10-15 degrees) was intended to release stretch on the $\mathrm{BB}$ muscle. However, a study on electrical stimulation of $\mathrm{BB}$ at different elbow angles showed that the optimal angle of biceps is about 20 degrees [37]. The angle of 10-15 degrees used may therefore still involve some stretch on the BB muscle, although the angle was not actually measured, so may have been near the optimal angle in cases. In light of the Koo et al. [37] study findings, which the current authors have become aware of since conducting the present study, the recommended elbow angle would be 20 degrees. An investigation is needed to document the effect of elbow angle on Myoton results within the range examined in the electrical stimulation study by Koo et al. [37]. Changes in the shape of the muscle with ageing may have caused variability in relation to testing the true muscle belly, which was more medial in older participants than experienced in younger participants in a related study [38]. The protocol could be modified to account for the likelihood that recordings were not reflective of the muscle belly, perhaps using a compromise between gaining a true midpoint along the length of the muscle, yet allowing an element of clinical judgment to ensure measurements are taken over the muscle belly. This could be achieved by visual observation and palpation, when asking the participant to make a gentle contraction of the muscle. The modified protocol has yet to be tested for reliability, both within sessions and between days.

\section{Potential clinical applications}

Accurate objective assessment of muscle tone in neurological conditions remains a challenge for clinicians. The MyotonPRO offers the ability to make accurate measurements rapidly, safely and reliably in the clinical setting. The frequency parameter measured, which reflects state of muscle tension, i.e. resting tone, can now be measured objectively but it may not be the most informative parameter in all situations. For example, greater muscle stiffness was found in patients with Parkinson's disease than in healthy controls [15], which might be expected from subjective clinical assessment. Much research is needed to determine the relative importance of the different parameters (tone, stiffness and elasticity) measured using the Myoton technology in different conditions. Muscle abnormalities can also be assessed in musculoskeletal and respiratory disorders. Potentially, both research and clinical practice could be greatly enhanced by using Myoton technology to aid assessment and monitor the effectiveness of pharmacological and physical interventions on skeletal muscles. Evidence from research is required before adopting such a tool in routine clinical practice and modifications to the technology could make it even more user friendly.

\section{Conclusions}

The present study has established the levels of between-side symmetry of tone and the mechanical properties of stiffness and elasticity of $\mathrm{BB}$ in healthy, community-dwelling older males, as measured using the MyotonPRO. Excellent within-session intrarater reliability was found. Measures of mechanical properties of skeletal muscle using hand-held devices, such as the Myoton PRO, would provide a potentially powerful objective tool in the stroke rehabilitation setting, as it is non-invasive, painless, safe, and relatively easy to use. The level of symmetry of BB may differ in younger age groups, in females, and in patients with different neurological conditions. Refinement of the testing procedure has been suggested and needs to be tested for reliability between days.

\section{Acknowledgements}

The authors thank the participants for their time, Dr Peter Nicholls for statistical advice, research colleagues on related projects Sandra Agyapong-Badu Lucy Aird, James Mullix and Kate Mooney for help when developing the protocol, Myoton Ltd (London) for loaning the MyotonPRO device and Aleko Peipsi (CEO, Myoton AS, Estonia) for providing training in its use.

\section{Conflict of Interest}

None to declare.

\section{References}

1. Tsivgoulis G, Spengos K, Manta P, Karandreas N, Zambelis T, et al. (2006) Validation of the ABCD score in identifying individuals at high early risk of stroke after a transient ischemic attack: a hospital-based case series study. Stroke 37: 2892-2897.

2. Lance JW (1980) The control of muscle tone, reflexes, and movement Robert Wartenberg Lecture. Neurology 30: 1303-1313.

3. Broeks JG, Lankhorst GJ, Rumping K, Prevo AJ (1999) The long-term outcome of arm function after stroke: results of a follow-up study. Disabil Rehabil 21: 357-364.

4. Bohannon RW, Smith MB (1987) Interrater reliability of a modified Ashworth scale of muscle spasticity. Phys Ther 67: 206-207.

5. Tardieu G, Shentoub S, Delarue R (1954) Research on a technic for measurement of spasticity. Rev Neurol (Paris) 91: 143-144.

6. Pandyan AD, Johnson GR, Price Cl, Curless RH, Barnes MP, et al. (1999) A review of the properties and limitations of the Ashworth and modified Ashworth Scales as measures of spasticity. Clin Rehabil 13: 373-383.

7. Tous-Fajardo J, Moras G, Rodríguez-Jiménez S, Usach R, Doutres DM, et al. (2010) Inter-rater reliability of muscle contractile property measurements using non-invasive tensiomyography. J Electromyogr Kinesiol 20: 761-766.

8. Horikawa M, Ebihara S, Sakai F, Akiyama M (1993) Non-invasive measurement method for hardness in muscular tissues. Med Biol Eng Comput 31: 623-627.

9. Aarrestad DD, Williams MD, Fehrer SC, Mikhailenok E, Leonard CT (2004) Intra- and interrater reliabilities of the Myotonometer when assessing the spastic condition of children with cerebral palsy. J Child Neurol 19: 894-901.

10. Leonard CT, Deshner WP, Romo JW, Suoja ES, Fehrer SC, et al. (2003) Myotonometer intra- and interrater reliabilities. Arch Phys Med Rehabil 84 928-932.

11. Koo TK, Cohen JH, Zheng $Y$ (2011) A mechano-acoustic indentor system for in vivo measurement of nonlinear elastic properties of soft tissue. J Manipulative Physiol Ther 34: 584-593.

12. Gavronski G, Veraksits A, Vasar E, Maaroos J (2007) Evaluation of viscoelastic parameters of the skeletal muscles in junior triathletes. Physiol Meas 28: 625-637.

13. Aird L, Samuel D, Stokes M (2012) Quadriceps muscle tone, elasticity and stiffness in older males: reliability and symmetry using the MyotonPRO. Arch Gerontol Geriatr 55: e31-39.

14. Mullix J, Warner M, Stokes M (2012) Testing muscle tone and mechanical properties of rectus femoris and biceps femoris using a novel hand held MyotonPRO device: relative ratios and reliability. Working Papers in the Health Sciences 1: 1-8.

15. Marusiak J, Kisiel-Sajewicz K, Jaskólska A, Jaskólski A (2010) Higher 
Citation: Bailey L, Samuel D, Warner M, Stokes M (2013) Parameters Representing Muscle Tone, Elasticity and Stiffness of Biceps Brachii in Healthy Older Males: Symmetry and Within-Session Reliability Using the MyotonPRO. J Neurol Disord 1: 116. doi:10.4172/2329-6895.1000116

muscle passive stiffness in Parkinson's disease patients than in controls measured by myotonometry. Arch Phys Med Rehabil 91: 800-802.

16. Chuang LL, Wu CY, Lin KC, Lur SY (2012) Quantitative mechanical properties of the relaxed biceps and triceps brachii muscles in patients with subacute stroke: a reliability study of the myoton- 3 myometer. Stroke Res Treat 2012: 617694

17. Chuang LL, Lin KC, Wu CY, Chang CW, Chen HC, et al. (2012) Relative and Absolute Reliabilities of the Myotonometric Measurements of Hemiparetic Arms in Patients With Stroke. Arch Phys Med Rehabil.

18. Bizzini M, Mannion AF (2003) Reliability of a new, hand-held device for assessing skeletal muscle stiffness. Clin Biomech (Bristol, Avon) 18: 459461.

19. Viir R, Laiho K, Kramarenko J, Mikkelsson M (2006) Repeatability of trapezius muscle tone assessment by a myometric method. J Mech Med Biol 6: 215-228.

20. Ditroilo M, Hunter AM, Haslam S, De Vito G (2011) The effectiveness of two novel techniques in establishing the mechanical and contractile responses of biceps femoris. Physiol Meas 32: 1315-1326.

21. Janecki D, Jarocka E, Jaskólska A, Marusiak J, Jaskólski A (2011) Muscle passive stiffness increases less after the second bout of eccentric exercise compared to the first bout. J Sci Med Sport 14: 338-343.

22. Roberts HC, Denison HJ, Martin HJ, Patel HP, Syddall H, et al. (2011) A review of the measurement of grip strength in clinical and epidemiological studies: towards a standardised approach. Age Ageing 40: 423-429.

23. Prado-Medeiros CL, Silva MP, Lessi GC, Alves MZ, Tannus A, et al. (2012) Muscle atrophy and functional deficits of knee extensors and flexors in people with chronic stroke. Phys Ther 92: 429-439.

24. Narici MV, Maffulli N (2010) Sarcopenia: characteristics, mechanisms and functional significance. Br Med Bull 95: 139-159.

25. Walter SD, Eliasziw M, Donner A (1998) Sample size and optimal designs for reliability studies. Stat Med 17: 101-110.

26. Oldfield RC (1971) The assessment and analysis of handedness: the Edinburgh inventory. Neuropsychologia 9: 97-113.

27. Washburn RA, Smith KW, Jette AM, Janney CA (1993) The Physical Activity
Scale for the Elderly (PASE): development and evaluation. J Clin Epidemiol 46: $153-162$

28. Gapeyeva H, Vain A (2008) Principles of applying Myoton in physica medicine and rehabilitation. Edited by Byrne A, Chen R, Devenny R, Forbes J: @ MÜOMEETRIA Ltd.

29. Fleiss J (2007) Reliability of measurement. In: The design and analysis of clinical experiments. New York, Wiley and Sons.

30. Portney L, Watkins M (2000) Statistical measures of reliability. In: Foundations of clinical research: Applications to practice Prentice-Hall Inc: Upper Saddle River, New Jersey.

31. Bland J, Altman D (1986) Statistical methods for assessing agreement between two methods of clinical measurement. Lancet 8: 307-310.

32. Desrosiers J, Bourbonnais D, Bravo G, Roy PM, Guay M (1996) Performance of the 'unaffected' upper extremity of elderly stroke patients. Stroke 27 1564-1570.

33. Gauthier J, Bourbonnais D, Filiatrault J, Gravel D, Arsenault AB (1992) Characterization of contralateral torques during static hip efforts in healthy subjects and subjects with hemiparesis. Brain 115: 1193-1207.

34. Nathan PW, Smith MC, Deacon P (1990) The corticospinal tracts in man Course and location of fibres at different segmental levels. Brain 113: 303 324.

35. Chuang LL, Wu CY, Lin KC (2012) Reliability, validity, and responsiveness of myotonometric measurement of muscle tone, elasticity, and stiffness in patients with stroke. Arch Phys Med Rehabil 93: 532-540.

36. Zinder SM, Padua DA (2011) Reliability, validity, and precision of a handheld myometer for assessing in vivo muscle stiffness. J Sport Rehabil Technical Notes 1.

37. Koo TK, Mak AF, Hung LK (2002) In vivo determination of subject-specific musculotendon parameters: applications to the prime elbow flexors in normal and hemiparetic subjects. Clin Biomech (Bristol, Avon) 17: 390-399.

38. Mooney K, Warner M, Stokes M (2013) Symmetry and within session reliability of mechanical properties of biceps brachii muscles in healthy young adult males using the MyotonPRO device. Working Papers in the Health Sciences (In press). 